\title{
Sovereign Debt Booms in Monetary Unions
}

\section{Citation}

Aguiar, Mark, Manuel Amador, Emmanuel Farhi, and Gita Gopinath. 2014. "Sovereign Debt Booms in Monetary Unions." American Economic Review 104, no. 5: 101-106.

\section{Published Version}

doi:10.1257/aer.104.5.101

\section{Permanent link}

http://nrs.harvard.edu/urn-3:HUL.InstRepos:12559514

\section{Terms of Use}

This article was downloaded from Harvard University's DASH repository, and is made available under the terms and conditions applicable to Open Access Policy Articles, as set forth at http:// nrs.harvard.edu/urn-3:HUL.InstRepos:dash.current.terms-of-use\#OAP

\section{Share Your Story}

The Harvard community has made this article openly available.

Please share how this access benefits you. Submit a story.

Accessibility 


\title{
Sovereign Debt Booms in Monetary Unions
}

\author{
By Mark Aguiar and Manuel Amador and Emmanuel Farhi and Gita Gopinath*
}

Your abstract here, please.

After joining the the euro, several countries with a history of high inflation (notable examples include Greece and Italy) experienced sharp reductions in inflation together with a prolonged build up of sovereign debt. In this paper we propose a rationalization for this phenomenon. To do so, we explore the interaction between inflation credibility and the debt dynamics that arise when an impatient sovereign issues nominal bonds. We are particularly interested in the impact of an increase in inflation credibility, achieved either through better policies and institutions or by leveraging the higher inflation credibility of other countries via a currency union. We show that an increase in inflation credibility delivers an invitation to borrow, raising the maximum borrowing limit of the country and reducing any incentive to save.

Conditional on a nominal interest rate, the government has an ex post incentive to inflate away the real value of its bonds. However, creditors anticipate this temptation ex ante, pricing the ex post inflation into the nominal interest rate. The country therefore bears the cost of high inflation with no reduction of debt in real terms. Including the costs associated with ex post inflation, a country with low inflation credibility will bear a greater burden from a given level of debt.

In such a scenario the government has an incentive to save and reduce debt over time to a level that eliminates the temptation to inflate. On the other hand, if the government is impatient relative to the market interest rate, it has an incentive to borrow and increase debt over time. This conflict between 'impatience' and 'saving to escape inflation' generates a cut-off level of debt below which the government will save and above which, it will borrow. That is, debt dynamics diverge around this cut-off, to the left of which debt shrinks and to the right of which debt grows.

One way to think about the above is through the elasticity of the nominal interest rate to debt levels. As the government accumulates debt, its nominal interest increases, reflecting the increase in the temptation to inflate. That positive elasticity can be high enough to stop the country from accumulating more debt at the margin, and thus it generates an endogenous force that breaks the borrowing dynamics generated by impatience.

When inflation credibility is high, and, as a result, the government is not tempted to inflate, then this elasticity force is reduced. As a result, the government borrows over time all the way up to its maximum debt limit. There is a range of debt levels over which the government will choose to save and reduce debt over time in a low inflation credibility regime but choose to borrow and increase debt over time in a high inflation credibility regime. That is, a switch from low to high inflation credibility can turn governments from savers to borrowers. ${ }^{1}$ In this sense we can rationalize why a country that gains the higher inflation commitment of a monetary union can end up with a sovereign borrowing boom, as was witnessed for some countries in the euro zone. ${ }^{2}$

* Aguiar: Department of Economics, Princeton University, Princeton NJ 08544 (maguiar@princeton.edu); Amador: Research Department, Federal Reserve Bank of Minneapolis, Minneapolis MN 55401 (amador.manuel@gmail.com); Farhi: Department of Economics, Harvard University, Cambridge MA 02138 (efarhi@fas.harvard.edu); Gopinath: Department of Economics, Harvard University, Cambridge MA 02138 (gopinath@harvard.edu). Amador acknowledges NSF support under award number 0952816. The views expressed herein are those of the authors and not necessarily those of the Federal Reserve Bank of Minneapolis or the Federal Reserve System. We thank Andy Atkeson for his comments.

${ }^{1}$ Importantly, inflation credibility is desirable from the perspective of the government. Indeed the sovereign is always better off when it has higher inflation credibility.

${ }^{2}$ More generally the interaction between inflation credibility and debt dynamics is important to understand why emerging markets historically borrowed exclusively in foreign currency, so called "original sin" (Eichengreen and Hausmann (2009)) and the more recent phenomenon of a decline in original sin as countries have managed to control inflation (Du and Schreger (2013)). Rebelo and Vegh (1995) also analyze the impact of inflation stabilizations on debt and output. 


\section{Environment}

The environment builds on the small open economy set up used in Aguiar et al. (2012) while introducing impatience to the government objective function. Time is continuous, the small open economy receives a constant endowment $y$ and issues nominal debt at interest rate $r_{t}$. The government chooses consumption and inflation and the price level evolves according to $P_{t}=P(t)=$ $P(0) e^{e_{0}^{t} \pi(t) d t}$, where $\pi(t)$ denotes the rate of inflation at time $t$. We assume $P(0)$ is pre-determined, and given the assumed continuity of $P(t)$ we can express the government's problem taking real debt $b(t)$ as the state variable.

We assume that the government lacks commitment, and analyze its decisions over time as a dynamic game. As in Aguiar et al. (2012), we focus attention on recursive equilibria, such that, when the government makes its optimal choices of inflation and consumption, it takes the equilibrium interest rate schedule, $r(b)$, as given. The maximization problem is then:

$$
V\left(b_{0}\right)=\max _{c(t), \pi(t)} \int_{0}^{\infty} e^{-\rho t}(u(c(t))-\psi \pi(t)) d t
$$

subject to:

$$
\begin{aligned}
& \dot{b}(t)=c(t)+(r(b(t))-\pi(t)) b(t)-y \\
& b(t) \in \bar{\Omega} \equiv\left[0, b_{\max }\right]
\end{aligned}
$$

where the set $\bar{\Omega}$ is to be determined in equilibrium. Note that we write the government's problem in sequence form, despite the lack of commitment to inflation policies. However, the government's problem is conditional on the equilibrium interest schedule $r(b)$; conditional on this schedule, the government has no conflict between inflation chosen as of $t=0$ and that chosen period-by-period.

The role of inflation as a partial default mechanism can be seen in the budget constraint expressed in real terms. The cost of inflation is modeled in reduced form as a deadweight welfare cost $\psi \pi(t)$. To keep the problem tractable we assume a linear inflation cost and restrict $\pi \in[0, \bar{\pi}]$.

While we do not micro-found preferences over inflation, a natural interpretation is that $\psi$ is a reduced-form proxy for a reputational cost to the government of inflation. A large cost represents an environment in which the government has a relatively strong incentive for (or commitment to) low inflation. T When performing comparative statics with respect to $\psi$, we have in mind institutional features of monetary and fiscal policy that vary across countries, such as the extent of inflation indexing in the private sector and the flexibility of prices; the political economy that governs the interaction of monetary and fiscal policy; the legislative mandate of the central bank and how readily this can be amended; and the ability to raise revenue through taxation in a non-distortionary manner.

Limited commitment manifests itself along two related dimensions. First, the government cannot commit to repaying its debt. If it defaults it enters autarky on a permanent basis and the welfare associated with autarky is $\underline{V}=u((1-\chi) y) / \rho$ where $\chi \leq 1$ captures any additional output costs associated with default. To ensure that the government has an incentive to repay its debt in equilibrium, it must be that $V(b) \geq \underline{V}$ for all $b \in \bar{\Omega}$. Given that $V$ will be monotone in debt, the equality $V\left(b_{\max }\right)=\underline{V}$ pins down the maximum debt limit $b_{\max }$ above which lenders will refuse to lend at any positive price. Second, the government cannot commit to an inflation and debt path, which is implicitly captured in the above problem by the fact that the government takes the interest rate schedule as given.

The lenders are assumed to be risk-neutral and in (a perfect foresight) equilibrium the interest rate schedule must satisfy the break-even condition:

$$
r(b)=r^{\star}+\Pi(b)
$$


where $r^{\star}$ is the real return on the outside option for the lenders and where $\Pi(b)$ is the equilibrium inflation policy (that is, the inflation policy that solves the governments problem above). We will assume that $r(b)$ satisfies the conditions of Definition 1 of Aguiar et al. (2012). In particular, in the equilibrium we will construct, $r(b)$ will have a point of discontinuity.

Assumption: $\boldsymbol{\rho}>\boldsymbol{r}^{\star}$. Differently from Aguiar et al. (2013), we assume that the government is impatient so that the discount rate exceeds the real interest rate $r^{\star}$ (the case of $\rho=r^{\star}$ is analyzed in Aguiar et al. (2012)). We can then define a Recursive Competitive Equilibrium exactly as in Definition 2 of Aguiar et al. (2013).

Let us now consider the solution to the government's problem (P1) for a given interest rate schedule. Define the following function:

$$
H(b, q)=\max _{\{c, \pi \in[0, \bar{\pi}]\}}\{u(c)-\psi \pi+q(c+(r(b)-\pi) b-y)\}
$$

The Hamilton-Jacobi-Bellman (HJB) equation is given by: $\rho V(b)-H\left(b, V^{\prime}(b)\right)=0$.

We face two problems when trying to solve the HJB equation. First, the value function itself is not necessarily differentiable, implying there may not be a classical solution to the HJB. And second, the interest rate schedule is not continuous, rendering the $H$ function discontinuous in $b$. However, in Aguiar et al. (2012) Definition 3, we show that the value function is the unique bounded continuous viscosity solution of the HJB, using the definition of viscosity solution of Bressan and Hong (2007). We will proceed in a similar fashion here.

As in Aguiar et al. (2012), we focus attention on monotone equilibria, and in particular, equilibria where the interest rate schedule is a step function. That is, for some $b_{\pi}$, to be determined below, the interest rate schedule is:

$$
r(b)= \begin{cases}r^{\star} & \text { for } b \leq b_{\pi}, \\ r^{\star}+\bar{\pi} & \text { for } b>b_{\pi},\end{cases}
$$

reflecting that equilibrium inflation, as a result of the linear inflation costs, will be a step function as well.

At points of differentiability of the value function and continuity of the interest rate schedule, the conditions in Definition 3 of Aguiar et al. (2013) boil down to the classical HJB equation. From there we can obtain the standard first order conditions:

$$
\begin{aligned}
u^{\prime}(c) & =-V^{\prime}(b) \\
\pi & =\left\{\begin{array}{l}
0 \text { if }-V^{\prime}(b) b<\psi \\
{[0, \bar{\pi}] \text { if }-V^{\prime}(b) b=\psi} \\
\bar{\pi} \text { if }-V^{\prime}(b) b>\psi
\end{array}\right.
\end{aligned}
$$

The first condition equates the marginal cost of debt to the negative of the marginal utility of consumption. The second condition states that inflation will be high when the marginal utility of consumption is high or debt is high. This reflects that the government has two ways of servicing nominal debt, saving and inflating. A high marginal utility of consumption (relative to $\psi$ ) favors inflating. Similarly, as the cost of inflating is independent of $b$ while the benefits are increasing, a high stock of nominal bonds favors inflating.

As discussed in Aguiar et al. (2012) there are in principle multiple values of $b_{\pi}$ that are consistent with equilibrium. As in that paper, we further narrow attention to the best monotone equilibrium, that is, the equilibrium with the largest $b_{\pi} \cdot{ }^{3}$ We proceed now to construct it.

\footnotetext{
${ }^{3}$ All other monotone equilibria can also be characterized in the way we do below.
} 


\section{Construction of the Equilibrium Solution: Low Inflation Credibility}

An equilibrium consists of an interest rate schedule that satisfies (L1) and a value function that is a viscosity solution to the HJB. As noted above, the candidate $r(b)$ is a step functions characterized by a threshold $b_{\pi}$ and a maximum debt level $b_{\max }$. Conditional on $\left\{b_{\pi}, b_{\max }\right\}$, the equilibrium value function is the unique viscosity solution to HJB. The uniqueness result discussed in Aguiar et al. (2012) is of particular value in this regard; namely, once we find a solution to the HJB, we know that it is the equilibrium value function associated with the candidate $r(b)$. We proceed by conjecturing an equilibrium and then verify it satisfies the HJB and the lenders' break-even conditions.

The conjectured equilibrium uses the following logic. For low enough values of debt, the government has no incentive to inflate and faces a low nominal interest rate. There is no reason for the government not to borrow, given its relative impatience. The question becomes more interesting at and above the threshold $b_{\pi}$. The higher nominal interest rate may discourage increasing debt beyond $b_{\pi}$, and conditional on $b>b_{\pi}$, may induce saving. We shall keep these issues in mind as we construct the value function.

We can construct a conjectured equilibrium starting with $b_{\pi}$. In particular, suppose the jump in interest rate at $b_{\pi}$ is sufficient that the government does not borrow at this threshold. In this case, $\dot{b}=0$ and the associated consumption is $C\left(b_{\pi}\right)=y-r^{\star} b_{\pi}$. For low inflation to be optimal, the first-order conditions require $u^{\prime}\left(y-r^{\star} b_{\pi}\right) b_{\pi} \leq \psi$. This expression evaluated at equality pins down the maximum $b_{\pi}$ that can be sustained conditional on $\dot{b}=0$. The associated value is $V\left(b_{\pi}\right)=u\left(y-r^{\star} b_{\pi}\right) / \rho$. For debt levels below $b_{\pi}$, the government will borrow up to the threshold. We can fill in the value function for $b<b_{\pi}$ using the HJB. In particular, the HJB implies that $V(b)$ satisfies an ordinary differential equation with the boundary condition $V\left(b_{\pi}\right)$. Note that the differential equation defines $V^{\prime}(b)$ implicitly. However, the strict convexity of $H\left(b, V^{\prime}\right)$ in $V^{\prime}$ implies that conditional on $V(b)$, there are only two possible values of $V^{\prime}(b)$ which satisfy the HJB, one which implies $\dot{b}>0$ and one which implies $\dot{b}<0$. For $b<b_{\pi}$, the "borrowing" solution is the appropriate $V^{\prime}(b)$.

For $b>b_{\pi}$, the government faces a choice. It can save in order to capture the low interest rate (and associated low inflation policy) that holds at $b_{\pi}$. Conversely, it can continue to borrow up to its maximum debt level. The value from saving can be obtained from the HJB extended rightward from $b_{\pi}$ under the conjecture $\Pi(b)=\bar{\pi}$ for $b>b_{\pi}$. That is, $V(b)$ satisfies the HJB with boundary condition $V\left(b_{\pi}\right)$, but now we follow the "saving" solution for $V^{\prime}(b)$. On the other hand, if the government were to borrow, it will accumulate debt up to $b_{\max }$. The value of this policy can be obtained by solving the HJB from $b_{\max }$. In particular, at $b_{\max }$, we have $\dot{b}=0$, as the government cannot exceed the borrowing limit. The associated value is $V\left(b_{\max }\right)=\frac{u\left(y-r^{\star} b_{\max }\right)-\psi \bar{\pi}}{\rho}$. As this is the borrowing limit at which the government is indifferent to repayment or default, we have $V\left(b_{\max }\right)=\underline{V}$, pinning down the value of $b_{\max }$ assuming $\dot{b}=0$. Given this boundary condition, the HJB can be solved to fill in the value for $b<b_{\max }$.

We therefore have two candidate values for each $b \in\left(b_{\pi}, b_{\max }\right)$, one associated with saving towards $b_{\pi}$ and the other associate with borrowing to $b_{\max }$. Let $b^{\star}$ denote the point at which they intersect. If such a point exists, then the optimal policy is to save for $b \in\left(b_{\pi}, b^{\star}\right]$ and borrow for $b>b^{\star}$. In what follows, we shall consider parameterizations that ensure that such a point exists. ${ }^{4}$

The conjectured value function links the segments defined over $\left[0, b_{\pi}\right],\left(b_{\pi}, b^{\star}\right],\left(b^{\star}, b_{\max }\right]$. The boundary conditions ensure that the proposed value function is continuous, and it solves the HJB at each point by construction. The solution must also satisfy the conjecture that $\Pi(b)=\bar{\pi}$ for

\footnotetext{
${ }^{4}$ More generally, we are characterizing an equilibrium such that $b_{\pi}$ and $b_{\max }$ are points of stationarity. Below we present a numerical parameterization in which this conjecture is verified. With other parameterizations, there exist other possible equilibria that can be found with the same algorithm. In particular, suppose that $b_{\pi}$ is a stationary point, but that it is optimal to save from $b_{\max }$ to $b_{\pi}$. In this case, the savings solution extended to the right of $b_{\pi}$ and evaluated at $b_{\max }$ lies above the steady state value. Similarly, if the borrowing solution extended to the left of $b_{\max }$ lies above the stationary value at $b_{\pi}$, then it is optimal to borrow to $b_{\max }$ from all points in $\bar{\Omega}$. In the interests of space, we do not discuss these alternatives further.
} 
$b>b_{\pi}$, which can be verified by checking $V^{\prime}(b) b \geq \psi$ at each point $b>b_{\pi}$. Note that the value function features two points of non-differentiability, $b_{\pi}$ and $b^{\star}$. It is at these points that the viscosity conditions must be verified. The resulting consumption policy will be discontinuous at those two points as well (see below in the numerical characterization). In particular, even though the government is more impatient than the foreign markets, the government is saving in a region to the right $b_{\pi}$ (that is, $\dot{b}<0$ for $\left(b_{\pi}, b^{\star}\right]$ ). This arises from the desire to reduce the equilibrium inflation rate. To see this, note that to the right of $b_{\pi}$, the government is choosing high inflation (consistent with the equilibrium). However, by saving just a bit, the government is able to bring the debt level down to $b_{\pi}$, a debt level consistent with zero inflation. By choosing to do this, in effect, the government is achieving commitment to low inflation by lowering its debt. Note that for $b$ sufficiently higher than $b_{\pi}$, the incentives switch: the government then gives up on the desire to commit to low inflation through savings (this is just too costly now), and instead just borrows up to the maximum level, while inflating along the way.

\section{Construction of the Equilibrium Solution: High Inflation Credibility}

We also construct an equilibrium when inflation credibility is so high that the government never inflates in equilibrium (that is, the value of $\psi$ is sufficiently high to rule out inflation for all equilibrium levels of debt). In this case, the equilibrium interest rate schedule will be just $r^{\star}$, and the HJB can be solved in the standard way. In this case $b_{\max }$ remains a stationary point, and thus $V\left(b_{\max }\right)=u\left(y-r^{\star} b_{\max }\right) / \rho$, and $b_{\max }$ is defined by $u\left(y-r^{\star} b_{\max }\right) / \rho=\underline{V}$. Note that this implies that the following condition $\psi>u^{\prime}\left(y-r^{\star} b_{\max }\right) b_{\max }$ represents a lower bound on $\psi$ in this case. Given this boundary condition, the value function satisfies the HJB such that $\dot{b}>0$ for $b<b_{\max }$.

Note that in this case, the country always borrows its way to the maximum debt possible. There is no savings region, and no other stationary value: the removal of the temptation to inflate also eliminates an endogenous force that puts a break on the desire to borrow to the maximum. Note as well that the amount of borrowing that can be sustained in this case, $b_{\max }$, is greater than when inflation credibility is low. This arises because inflation credibility reduces the incentive to default, as debt is no longer associated with high equilibrium inflation. In the next section, we compare numerical solutions to the HJB in the two cases above for parameters such that the solutions just described exist.

\section{Inflation credibility}

The left panel of Figure 1 compares the consumption policy function for the case when inflation credibility is low, $\psi_{1}$, to that when inflation credibility is high $\psi_{2}$.

In the case of $\psi_{2}$, inflation costs are high enough so that inflation is throughout zero. In the case of $\psi_{1}$, inflation costs are low enough that $b_{\pi}<b_{\max }$ exists. As discussed previously, the consumption policy function for $\psi_{1}$ is then characterized by a region of borrowing, $0<b<b_{\pi, \psi_{1}}$, followed by a region of saving $b_{\pi, \psi_{1}}<b<b_{\psi_{1}}^{\star}$ and then another region of borrowing $b_{\psi_{1}}^{\star}<b<b_{\text {max, } \psi_{1}}$, as marked in the left panel of Figure 1.

Also as discussed previously, for $\psi_{2}$ since there is no incentive to save, consumption is forever decreasing and debt is forever increasing until the debt limit is reached.

There are two interesting features to this comparison. The debt limit for $\psi_{2}$ exceeds that for $\psi_{1}$ and in the region $b_{\pi, \psi_{1}}<b<b_{\psi_{1}}^{\star}$ the country saves in the low inflation cost environment while borrowing in the high inflation cost environment. The value functions are depicted in the right panel of Figure 1. As can be seen, the country is always better off in a high inflation cost versus low inflation cost environment. 

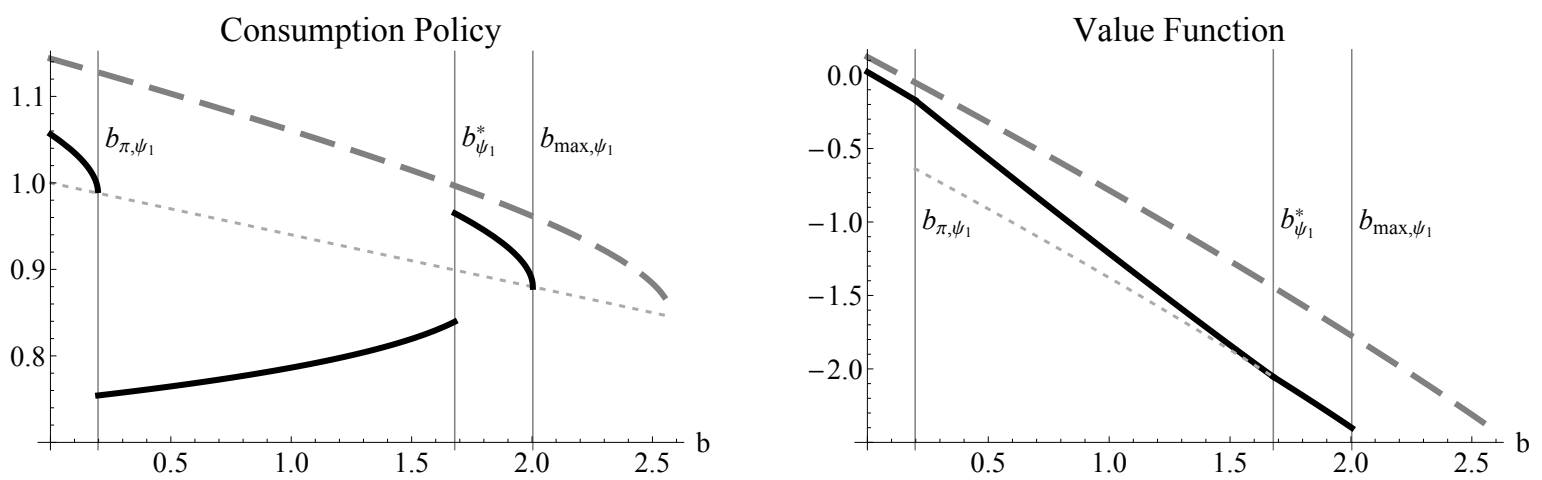

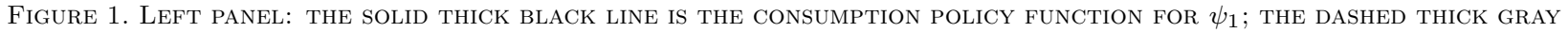
LINE IS THE CONSUMPTION POLICY FUNCTION FOR $\psi_{2}$; AND THE DOTTED THIN LINE IS THE STATIONARY CONSUMPTION VALUE,

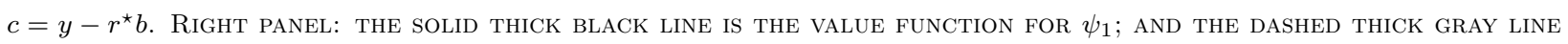
is the value function for $\psi_{2}$. The parameters in Both panels are $u=\log , r=0.06, \rho=0.07, y=1, \psi_{1}=0.2, \bar{\pi}=0.2$, $\chi=0.1547$, AND $\psi_{2}$ SUFFICIENTLY HIGH.

\section{Conclusion}

In this paper we explore the interaction between inflation credibility and impatience in determining debt dynamics and debt limits. We show that this interaction can rationalize why a country that attains higher inflation credibility by joining a monetary union ${ }^{5}$, all else equal, can experience a build up of sovereign debt as has been documented for countries like Greece on joining the euro.

\section{REFERENCES}

Aguiar, Mark, Manuel Amador, Emmanuel Farhi, and Gita Gopinath. 2012. "Crisis and Commitment: Inflation Credibility and the Vulnerability to Sovereign Debt Crises." working paper.

Aguiar, Mark, Manuel Amador, Emmanuel Farhi, and Gita Gopinath. 2013. "Coordination and Crisis in a Currency Union." working paper.

Bressan, Alberto, and Yunho Hong. 2007. "Optimal Control Problems on Stratified Domains." Networks and Heterogeneous Media, 2: 313-331.

Du, Wenxin, and Jesse Schreger. 2013. "Local Currency Sovereign Risk." working paper.

Eichengreen, Barry, and Ricardo Hausmann. 2009. Other People's Money: Debt Denomination and Financial Instability in Emerging Market Economies, The University of Chicago Press, Chicago and London (2005). Chicago, IL:The University of Chicago Press.

Rebelo, Sergio, and Carlos A. Vegh. 1995. "Real Effects of Exchange Rate-Based Stabilization: An Analysis of Competing Theories." National Bureau of Economic Research, Inc NBER Working Papers 5197.

\footnotetext{
${ }^{5}$ For an analysis of the interaction between debt and inflation policy in a monetary union subject to self-fulfilling crisis see Aguiar et al. (2013).
} 